not agree with Baruch in his sweeping statement that "every indication is fully met by the bath." But this point I wish to emphasize, if used judiciously in conjunction with other remedies its value is of unmeasured worth.

Abuses and contra-indications.-It is certainly a fact that one disastrous case from cold bathing will do and indeed has done, more toward creating a feeling of antagonism to the method than 1,000 cases of undoubted success will do toward forwarding it; and that, in spite of the fact that it was done ignorantly, or in direct disregard of all physiologic laws. Baruch mentions a case in point. A young hospital physician and teacher expressed abhorence of the cold bath treatment of typhoid; and when asked why, he said he had seen a nurse killed by it, and this is the description of their method as given by him: "We endeavored to reduce her temperature by wrapping her in a sheet, placing her on a Kilbs cot, and sprinkling her with ice water." Do you wonder that it proved to be her winding sheet? And Baruch gives another in. stance of the same method being used in one of the hospitals of a large city. Another way in which the cold bath is brought into ill repute is by not exercising the proper amount of care in selecting the cases for baths. Patients with atheromatous vessels, with organic heart disease, with hearts which are already on the verge of disolution from some exhausting disease, should never be put in the cold bath. Indeed, 1 am of opinion that there are some nervous organisms which can not stand the cold plunge even in health. lacking the one desideratum, namely, the abil. ity to react quickly and completely. While mentioning the abuses of the cold bath in therapeutics, it will not be out of place to give a very striking instance of the extent to which their fervor will carry some in using it as a hygienic measure. I was told by a young man who had been in the habit of taking a cold bath every morning, that he was in the country and arising early and having no means handy for taking his accustomed cold bath, he went down to the river. and breaking a hole in the ice, jumped in. He was fortunate to escape serious results; but it demonstrates the folly of carrying any measure to extremes. The one cardinal point always to be borne in mind in health and disease is to get reaction.

And now in closing let me emphasize the point, that although the cold bath is a great aid in the batthe against disease, it is not a specific for the germ disease, nor in fact for any disease. It is only one of the agents to combat the symptoms, but a powerful one. And if used rationally, understandingly, conscientiously, I think you will agree with me in giving it it rightful place among the foremost therapeutic agents of the day.

The Cairo Flats.

\section{THE URINE IN DIABETES MELLITUS.}

BY ARTHUR R. ELLIOTT, C.M., M.D.

PROFESSOR OF PREVENTIVE MEDICINEAND URINARY DIACNOSIS AT THF POST GRADUATE MEDICAL SCHOOL, CHICAGO.

While diabetes mellitus is not a disease of the kidney, it finds its chief clinical expression in certain profound alterations in the character of the urine. This fact renders the study of that secretion of primary importance in the diagnosis and management of the disease. The great practical value of urinary analysis in these cases is generally recognized, but the importance of painstaking and frequently repeated examinations is not so extensively appreciated. An analysis which includes only the detection of sugar and the estimation of the specific gravity and reaction is rudimentary and neglects many valuable considerations. Close observation of the urine will help us to a more perfect understanding of the prugress of the case and its prognosis, and will result in the early detection of developing complications.

I have been impressed by the comparatively casual manner with which the urinary changes of this affection are considered in special and general text-books, and the following detuiled description of the urine in this interesting condition is inspired thereby. In no disease are the urinary changes more profound. Hardly a single character, physical or chemic, escapes some alteration. Most marked are those affecting the physical characters of the urine. Usually the first symptom to attract the attention of the patient is the polyuria. Impressionable and nervous patients become immediately much alarmed on this account. The quantity of urine in nearly every case of true diabetes mellitus is greatly increased, the average range being from eight to fifteen pints daily. It has been known to exceed thirty-two pints, and Bence Jones reported a case in which the daily excretion reached seven gallons. The quantity, while constantly increased, varies greatly from day to day and at different times of the day. It bears a pretty constant relation to the excretion of sugar, the polyuria diminishing with improvement in the glycosuria and consequent diminution of thirst. No more water is excreted by the kidneys than is ingested, as was formerly alleged. Polyuria is une of the most constant urinary signs of diabetes, and if a high specific gravity be associated with it, is of considerable diagnostic importance. In rare instances the disease is not attended by polyuria. Frank has named this exceptiunal form "diabetes decipiens." Such cases are generally considered less serious than those in which the urine is abundant. The color of diabetic urine depends much upon the degree of ailution. Typical diabetic urine is a pale amber, but when not attended by marked polyaria it may be of dark color. When heavily charged with sugar it may have a greenish hue. The quantity of urine secreted during the night is as a rule less than during the day, contrary to the general rule in pathologically induced diuresis. Diabetes is often ushered in by a pre-glycosuric diuresis lusting for a considerable time. Likewise a post-glycosuric polyuria attends convalescence when a cure of the disease takes place. This latter is probably accounted for by the polydipsia which persists for a considerable period after the sugar has disappeared from the urine.

The urine in this affection constitutes a marked exception to the general rule that increase in quantity is attended by a diminution of specific gravity, for notwithstanding its large volume, the density is high. This is due to the presence of sugar and to the augmented excretion of urinary solids. The specific gravity has been noted as high as 1070, although the average range is between 1028 and 1045. It is not invariably increased, however. Saunby, Flint, Cameron and others have reported cases of undoubted diabetes mellitus attended with low specific gravity. Such cases are rare, and when met with should be regarded with suspicion, appeal being made to the phenylhydrazin or fermentation tests to prevent error, as faulty reduction of the cupric oxid tests by non- 
saccharine reducing substances may have taken place. In addition the urine should be submitted to careful microscopic exumination for casts and be tested for -albumin, as an incipient interstitial nephritis may coexist.

Urine heavily charged with sugar may have a diminished consistence. When shaken such urine forms an abundant froth. This does not subside as quickly as does that of normal urine, but is less creamy and permanent than the froth of highly albuminous urine.

The transparency is not usually altered. Indeed, in this condition the urine is notably clear and free from sediment, as a rule. Occasionally, however, and most frequently is this the case in gouty subjects, the urates may be precipitated. Upon standing, the urine may assume a bright and opalescent hue, and if exposed to warm air for a time, a white flour-like deposit of yeast fungus forms in the bottom of the vessel. If indican be present in excess, as it frequently is in this condition, the urine becomes red upon standing, from oxidation of that product. Blood has been noted as a deposit in advanced cases.

The odor of diabetic urine frequently resembles that of hay, but in advanced cases may possess the peculiar chloroformic order of acetone. This often precedes the development of diabetic coma and when present should excite apprehension. The urine has a distinctly sweet taste from the presence of sugar. In certain rare cases air is voided with the urine. This pneumaturia arises from a formation of gases in the bladder consequent upon fermentation changes in the urine.

The chemic reaction is invariably acid unless complications exist, and, as a rule, more sharply so than in health. This acidity persists apparently undiminished during the "alkalin tide" after meals, and is not affected to any degree by the administration of alkalin remedies. Upon standing the urine becomes more acid, alkalin fermentation being long delayed. On the other hand, alcoholic fermentation quickly supervenes, the specific gravity at the same time being lowered from the conversion of sugar into alcohol. Derignac has pointed out that the acidity is increased with the acetonemia of diabetic coma. The total daily excretion of urinary solids is increased.

A brief glance at the condition of the normal urinary constituents will develop some important and significant facts. The elimination of urea is almost invariably increased, and this is the case throughout the disease. Occasionally we notice a fall in the excretion of urea without assignable cause, and such an event frequently presages diabetic coma. The increase of urea is brought about by a variety of causes. They are briefly, 1 , largely increased ingestion of nitrogenous food rendered necessary by the anti-diabetic dietary; 2 , increased tissue metamorphosis; 3 , great functional activity of the liver; 4 , to some extent to the washing out of the tissues by the diuresis. This increase in urea bears a direct relation to the severity of the disease.

Other urinary solids are also affected, although to a less extent. The excretion of uric acid is slightly increased as a rule, although it is seldom seen as a deposit. The reason for this is that the largely diluted urine exercises an increased solvent power in proportion to its dilution, and keeps the uric acid in solution when it would otherwise appear as a deposit. Occasionally, in gouty subjects, a precipitate of urates is observed. Frequently an improvement in the patient's condition and a diminution of the polyuria is attended by a deposition of urates and uric acid crystals.

The inorganic constituents of the urine, the chlorids, phosphates and sulphates, are as a rule somewhat augmented. This is partly due to the diet and partly to the washing out of the tissues. It is well to bear in mind that this increase in the urinary solids is not a relative one, but an absolute increase. The large dilution of the urine may result in their proportionate diminution, while in the aggregate they are increased. Indican is found in excess in diabetic urine, and when exposed to the air such urine becomes deeply red. The increase is greatest just before and during coma, probably the result of the constipation and intestinal fermentation accompanying that condition. According to Stadelmann, Von Norden, Wolpe and others, ammonia is frequently present in large amount, but is neutralized by the beta-oxybutyric acid present and the urine remains acid. An increase in hippuric acid and creatinin has also been noted.

While the foregoing facts are important clinically, it is upon the presence of sugar in the urine that the diagnosis rests. The mere presence of sugar or copper-reducing substance will not suffice for a diagnosis of diabetes mellitus. It is necessary to establish beyond doubt its presence as a constant product in the urine, and also the fact of its excretion in some considerable quantity in order to distinguish from other and less serious forms of glycosuria. The average range of sugar excreted is over 4 per cent. not uncommonly rising to 7 or 8 , or even 12 per cent. The total daily excretion may be but a few drams or may reach a pound or more. It fluctuates greatly from day to day, varying with the diet and physical condition. An intercurrent attack of fever or some grave disturbance of the general system will sometimes result in a diminution or total disappearance of the sugar for a time. It is also diminished before and during diabetic coma. Starchy foods, mental worry, over-exertion, biliousness, are among the causes which increase the glycosuria.

The tests most commonly used for the detection of sugar in the urine are the cupric oxid tests, and of these Fehling's method has been for years the one most generally employed. In common with the others of this class, it depends for its reaction upon the power which glucose possesses of reducing cupric oxid to lower forms of oxidation with the formation of a yellowish or red precipitate soluble in ammonia. For the detection of large quantities of sugar in the urine, Fehling's test is a reliable method. When, however, but a small quantity is present, its results can not be depended upon. In its application we are directed to add the urine to the boiling test drop by drop until reduction takes place or until as much urine has been added as we have test solution. The quantity of solution emploved is usually one dram.

There are certain non-saccharin substances present in the urine which reduce cupric test solutions, and they constitute a fertile source of error when but slight reduction takes place. Of these substances the most misleading, because present in normal urine, are uric acid and kreatinin. With Fehling's solution concentrated normal urine will almost invariably produce a reaction closely resembling that due to small quantities of sugar. This pseudo-reduction is owing to the presence of these two substances in excess, their proportions being raised by the concentration of the 
urine. The large quantity of urine used in the application of Fehling's test renders it very susceptible to this error. If a smaller quantity be used its delicacy is impaired. It is with urines of high specific gravity and increased acidity that we exercise the greatest care in testing for sugar. A high specific gravity does not by any means always indicate the presence of sugar, for very frequently we see concentrated urine with a density of 1025 to 1032 which contains no sugar. Such urine produces false reduction of Fehling's test solution, consequently in cases where discrimination is essential this method is most unreliable and misleading. Glycuronic acid is another possible cause of error. It is present in excess in the urine after the ingestion of camphor, chloral and chloroform, and gives rise to a reduction of the copper tests closely resembling that produced by glucose. The smaller the quantity of urine that is used in testing the less likely is this mistake to occur. In addition to these sources of error in using Fehling's test, its instability, the influence which the presence of albumin has in interfering with the reduction, and its well-known tendency under certain circumstances to undergo spontaneous reduction, are other objections to its use.

It is important to be able to recognize minute traces of sugar in the urine, for while such may give rise to no appreciable symptoms, they are indicative of a tendency which may at any time, unless corrected, develop into diabetes mellitus. This can not be accomplished with certainty by Fehling's method for reasons already recited. In striving to accomplish this end and at the same time overcome the disadvantages of the methods in common use, I have evolved the following method, ${ }^{1}$ which has thus far in my hands given most satisfuctory results. It is delicate, recognizing with certainty as small an amount of sugar as .1 per cent, while requiring the employment of but a very small quantity ( 8 drops) of urine, thus possessing the two great essentials of a perfect test for sugar. In addition it is simple, easily applied and accurate, and its reaction, even with the smaller quantities of sugar, is easily distinguishable. It is prepared and applied as follows. Solution No. 1 :

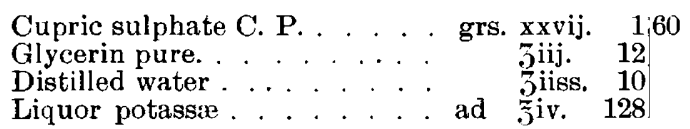

Dissolve the copper sulphate in the glycerin and water. Gentle heat will facilitate its solution. When cold add the liquor potassæ, mix thoroughly and filter.

Solution No. 2 is a saturated solution of chemically pure tartaric acid in distilled water.

These solutions, when carefully prepared, are quite stable and with ordinary care will keep unimpaired for months.

Into a test tube pour one dram of the cupric oxid solution (No.1) and gently boil over a spirit flame. Then add two or three drops-never more-of the tartaric acid solution and boil again. The solution remains perfectly clear and transparent and is of a beautiful blue color. Now add the suspected urine drop by drop, boiling and shaking the test between each drop until reduction takes place or until eight drops of urine have been added. If no reduction follows the addition of this amount of urine, sugar is not present. The end reaction is a yellowish-red deposit of cuprous oxid which is marked and unmistakable.

\footnotetext{
Jew York Medical Journal, July 27, 1895.
}

The reaction deepens when allowed to stand for a few moments.

Applied in this manner this test will detect one part in 1,000 of urine, or less than one-half grain to the ounce. If the percentage of sugar be large, a single drop of urine will promptly develop the reaction. The addition of three drops gives a marked reduction with two grains, and four drops will detect one grain to the ounce of urine. In applying the test care must be taken not to use a larger quantity of the tartaric acid solution than three drops to the dram of copper solution. If more than this be used the character of the reaction is affected, a discoloration rather than a precipitate being produced, and the delicacy of the test is also somewhat impaired. A greater quantity of urine than eight drops should not be used. By so doing greater delicacy may be obtained, but reliability will be sacrificed for increased sensitiveness, and the special value of the method destroyed. The presence of albumin in the urine does not act as a deterrent to this reaction.

Glycuronic acid, when present, gives rise to considerable confusion in testing for sugar. It is found in normal urine in such small quantity as to be considered practically absent, but after the administration of certain drugs it may appear in considerable amount, in which event it throws down a heavy precipitate with copper test solutions, indistinguishable from the reduction produced by grape sugar. This condition was erroneously considered a form of glycosuria by the older observers. If its presence misleads, certain discrepancies in the urine such as low specific gravity, absence of polyuria, together with absence of the physical symptoms of diabetes and a history of the taking of one of the drugs mentioned, will excite suspicion of error, and an appeal to the fermentation or phenylhydrazin test will clear away all doubt. The latter is the most reliable qualitative test for sugar, as that substance is the only urinary product which produces the characteristic reaction. It is an excellent appeal test when the indication of the copper reduction method is doubtful. Its application, however, consumes some time, and the phenylhydrazin is inconvenient to handle and very irritating to the skin. Havelburg's modification will be found much easier to carry out than the original method of Von Jaksch. It is applied as follows. Place 20 grs. (gm. 1.20) of phenylhydrazin hydrochlorate and 30 grains (gm. 1.80) sodium acetate in a test tube and fill almost half full of distilled water and slightly warm. An equal quantity of the urine is now added and finally a little chloroform, and the mixture shaken. If the test tube be allowed to stand the chloroform soon settles to the bottom, and if sugar be present a layer of fluid forms above it, containing the crystals of phenylglucosazone. Some of this layer of fluid is gently withdrawn by a pipette and mounted on a slide, and examined under the microscope for the characteristic crystals.

Having established the fact of the presence of sugar in the urine it remains to determine its quantity. This should be invariably practiced in cases where sugar is found in the urine, for by the knowledge so gained we are assisted to a definite understanding of the disease and its prognosis. Repeated estimations will enable us to closely watch the progress of the case and observe the effects of treatment. It is not now the un. satisfactory procedure that it once was. Modern inethods have done much to simplify the process and now with inexpensive apparatus and the expenditure 
of a few minutes of time the knowledge so essential to the conduct of the case can be gained.

The methods most used are the fermentation method, optical saccharimetry. Fehling's method th by titration and the ammoniated cupric tests, which include Pavy's and Purdy's methods. I have adapted my own method previously described for the detection of sugar so that it may be employed for quantitative estimation after the manner of the ammoniated cupric tests. A comparison of the different methods just enumerated will not fail to demonstrate the superiority of this class of tests. As they require but a few minutes for their application the estimation may be made without delay, indeed while the patient waits, an important practical advantage which the others do not possess. Furthermore the results are accurate, uniform and reliable.

The advantages which characterize my method as a qualitative test render it also especially applicable for quantitative estimation. It is a test which is easily applied and possesses a singularly clear and transparent end reaction. The principle of the method in common with the others of the ammoniated cupric class is that the cuprous oxid formed by the reducing power of the sugar is held in solution by ammonia which is added for that purpose, the test remaining clear and transparent throughout, the end reaction being the complete disappearance of the blue color.

The test is employed by taking 133 minims of the cupric oxid solution (No. 1) in a narrow necked glass flask and adding thereto 6 drops of the tartaric acid solution and 3 drams (cc. 12.) of liq. am. U.S.P. Mix thoroughly and add distilled water to raise the total volume to two fluid ounces (cc. 64). The resulting solution is perfectly clear and transparent. This amount of test solution so prepared represents in sugar value one-fourth grain of grape sugar, that is, it is reduced and decolorized by exactly that amount of sugar. 'The necessary apparatus is a glass flask with narrow neck, a retort stand, alcohol lamp and minim pipette. The reagent prepared as described is brought to the boiling point over a spirit flame and while smartly boiling, the urine is added from the pipette slowly drop by drop alluwing a few seconds to intervene between each drop until the blue color has entirely disappeared from the test solution. As the color fades it is well to proceed more slowly with the addition of the urine. The addition must be stopped immediately the color has entirely disappeared and the number of minims employed should be noted. To facilitate observation of the progress of decoloration a white background may be used. If now 480 minims (cc. 32 ) be divided by the number of minims required to decolorize the test and the quotient be divided by 4 we obtain the number of grains of sugar to the ounce of urine. Knowing the total excretion of urine it is a simple matter to estimate the total loss of sugar for the twenty-four hours. If the urine be heavily charged with sugar greater accuracy may be obtained by diluting it before testing with 2 volumes of distilled water and multiplying the result by 3 . There is no difficulty in determining the exact point of complete reduction. After decoloration the solution is clear and transparent with a slightly opalescent hue. In clearness of reaction this test excels all others of its class. This is due partly to its extreme delicacy and also to some extent to the fact that it is freshly prepared for each application. Notwithstanding their alleged stability the other tests deteriorate to some extent after being kept for a length of time and this detracts from the clearness of their end reaction. It is well to bear in mind the following precautions in applying this test. If the urine be dropped in too slowly and the boiling is thus much prolonged some suboxid may be thrown down because of evaporation of the ammonia before decoloration is complete. The test solution should be kept steadily boiling until reduction and decoloration are complete, as any interruption of ebulition will permit the entrance of air to the solution, thus producing possibly some reoxidation of the reduced oxid, which would render the result slightly fallacious. These accidents with a little care can be readily guarded against. Upon standing after decoloration the blue color gradually returns to the solution. This is owing to absorption of oxygen from the air and the reformation of the blue protoxid from the suboxid held in solution by the ammonia. The accuracy of the results of this method may be verified by the employment of a solution of known strength of chemically pure grape sugar in distilled water or normal urine.

Microscopically there is nothing characteristic in the urine of diabetes mellitus unless it be the presence of yeast plants (saccharomyces urinæ). These little cells when present may be takan as positive evidence of the presence of sugar. I have frequently observed that highly saccharin urine is very prolific in mucus cylindroids. These are due no doubt to irritation of the mucous surfaces by the highly acid abnormal urine.

In view of the frequent development of interstitial changes in the kidneys during the progress of diabetes and the important bearing which such have upon the prognosis it is well from time to time to submit the urine sediment to careful microscopic inspection for casts. The frequent association of casts and diabetic coma would furnish other reasons for care in this direction. The sediment of diabetic urine is as a rule very sparse. The high density of such urine greatly delays the deposition of the light hyaline casts in the sediment and thus renders their detection often very difficult. Since the introduction of the centrifuge into urinary work this difficulty has been overcome. In the sediment obtained by centrifugal force these casts if present can always be detected.

The irritation of the urinary passages by the highly acid abnormal urine and the lowered vitality and powers of resistance always present in these cases, result in the frequent occurrence of suppuration at some point of the urinary canal. If such be present the microscope will reveal the characteristic pus and epithelial elements.

Not the least among the advantages to be derived from analysis of the urine in this disease is the discovery of certain conditions which may develop during its course, which have an important bearing upon prognosis and treatment. For this reason frequent pains-taking uranalyses should be made; for by their early detection we are often warned of impending danger and may be enabled by instituting prompt measures to evert or postpone some grave complication. In this manner a sudden fall in specific gravity and the excretion of sugar and urea without corresponding amelioration of the physical condition should be regarded with apprehension as these phenomena frequently preceed diabetic coma. The urine should be at once submitted to the ferric chlorid test. Of the significance of this reaction Halliburton says: "What- 
ever the substance may be that causes the reaction, its appearance is of grave import often foretelling the onset of coma and death." According to this authority ethyl-diacetic acid is the cause of the reaction. Von Jaksch also considers that it often forebodes diabetic coma, and Bouchard in his valuable work on "Auto-intoxication" ascribes to it considerable value as a prognostic sign. He says: "In nearly all cases of diabetic coma, perhaps in all, we find this color reaction of the urine. . . . In diabetes this reaction may serve as a basis of diagnostic and prognostic indication." Bouchard states his belief that the substances producing this reaction are the result of defective elaboration of matter by the human organism and are largely formed in the intestine. Among these substance are acetone, diacetic acid and oxybutyric acid, all of which are poisonous. From these statements we might expect that careful regulation of the diet and a course of intestinal antiseptics and digestants with measures to combat constipation if instituted early in the disease and persistently maintained would have a decided effect in postponing this fatal complication. With this color reaction, a fruity chloroformic odor of the breath and urine, $\mathrm{kn}$ excess of indican and an increase in acidity of the urine are frequent accom. paniments.

The appearance of renal casts is also significant of developing coma. In twenty cases of diabetic coma Sandmeyer found numerous casts in every instance. They were present both during the prodromal symptoms and when the coma was marked. Kulz more recently in observations conducted in 400 cases likewise found casts both before and during coma. No difference was noted in their number whether the coma terminated fatally or in temporary recovery. The latter event was followed by disappearance of the casts from the urine. A small amount of albumin was frequently but not invariably present. The appearance of casts would seem to be a prognostic sign of value in these cases. In this connection care must be exercised not to mistake for casts the cylindroids so commonly present in diabetic urine and which often bear a strong resemblance to light hyaline casts. It is also well to bear in mind the fact that casts are frequently present in the urine of diabetics due to interstitial changes in the kidneys brought about by prolonged irritation. Prognostic significance can only be attached to their appearance when the urine has previously been quite free from them.

Albuminuria is a symptom of frequent occurrence in diabetes and is therefore worthy of attention. Bouchard has pointed out that it is present in 43 per cent. of cases. Smitz found that in 1300 diabetics 824 were also subject to albuminuria. Murie alleges that such cases are principally arthritic diabetics. The albuminuria is usually slight, frequently but a trace of albumin being present, or it may reach one gram in the twenty-four hours, beyond which amount however it seldom goes unless complications exist. The prognostic importance of this sign depends upon the grade of the albuminuria and more especially upon the association of symptoms of Bright's disease. If the latter be associated with it the prognosis is grave. The following are some of the most frequent causes of albuminuria in diabetes:

1. Irritation by the sugar during its excretion, bringing about degenerative changes of a hyuline character in the small arteries and the epithelium of the renal tubules,
2. Suppurative conditions of the urinary passages to which diabetes are so predisposed.

3. The largely increased ingestion of albuminoid foods.

4. The increased liability of diabetics to various morbid changes because of poor nutrition and lowered resistence.

5. Derived albumin the result of dissimilation.

6. A coëxisting nephritis.

In rare instances the albuminuria and glycosuria may alternate. These cases Sir Dyce Duckworth considers of a gouty nature. The albuminuria has been known to substitute the glycosuria, sugar disappearing entirely from the urine. When albumin is present a microscopic examination should invariably be made. Occasionally a case is met with in which the degenerative changes in the kidney progress and assume the ascendency. The nephritis becomes in time the more threatening condition and may eventually prove fatal. In their management these cases present many difficulties to the physician, an exceedingly nice adjustment of the diet being necessary to hold in check the two diseases. Disappearance or marked diminution of the glycosuria upon withdrawal of starches from the diet is a favorable indication.

Lastly the longer the glycosuria persists the more unfavorable is the progress.

Auditorium Hotel.

\section{SERUM THERAPY IN TETANUS-WITH CLINICAL REPORT. \\ BY T. A. DEWAR, M.D. DETROIT. мICH.}

August 2, I was asked to see a boy suffering with a sore neck and back, which his father regarded as being myalgic in nature. On arriving at the bedside at $10 \mathrm{~A}$. M. my attention was at first attracted to a sore foot protruding from beneath the bedelothes. On inspection the wound proved to be an ugly laceration on the sole, over the base of the fifth metatarsal bone, which was carefully sealed up with court plaster, care being taken that none of the pus should escape. I at once tore off the plaster and pushed my thumb deeply through the foul wound, preparatory to sterilization. While scraping out the wound with my thumb the boy, to my surprise, suddenly assumed the position of opisthotonos, no part of the body touching the bed except his heels and the back of the head, the body being arched almost to the extent of a semicircle. I recognized the clinical picture of tetanus; tetanic spasms, spastic masseters with trismus, retraction of the head, the risus sardonicus, rigidity of the cervical, dorsal and lumbar muscles, with intense constitutional disturbance; pulse 135, respiration 21 , temperature 100 degrees $F$.

I at once stayed proceedings and took his history, which I present as follows: H. McD., male, age 11, family and personal history excellent. July 24, patient stepped on some broken glass, receiving the lacerated wound described above. No attention was paid to it, except to apply court plaster, and patient made no complaint and appeared as well as usual till July 31 , when slight impairment of mastication and deglutition was noticed. The following day he became progressively worse and complained repeatedly of intense stiffness of his neck and back, which in fact were in a spastic condition. At this time mastication was impossible and deglutition very dangerous. 